\section{PWE-090 MANAGEMENT AND SHORT TERM OUTCOMES OF MALIGNANT COLORECTAL POLYPS IN THE NORTH OF ENGLAND}

doi:10.1136/gutjnl-2012-302514d.90

${ }^{1} \mathrm{M}$ D Gill, ${ }^{*}{ }^{2} \mathrm{M}$ D Rutter, ${ }^{3} \mathrm{~S} J$ Holtham. ${ }^{1}$ Northern Colorectal Cancer Audit Group, Northern England; ${ }^{2}$ University of Durham, Durham, UK; ${ }^{3}$ Department of General Surgery, Sunderland Royal Hospital, Sunderland, UK

Introduction Management of malignant colorectal polyps (MCPs) is contentious with no randomised controlled trials comparing endoscopic with surgical management. This study reviews the management and outcomes of MCPs across a UK region.

Methods Patients were identified using the NORCCAG (NORthern Colorectal Cancer Audit Group) database between April 2006 and July 2010. All histopathology reports and follow-up procedures were reviewed.

Results Of 386 patients identified, 165 (42.7\%) had the polyp biopsied, $37(9.6 \%)$ a piecemeal excision, $184(47.7 \%)$ a polypectomy. All initial biopsies underwent surgical intervention. 103/221 initial local excisions (46.6\%) had follow-up surgery of whom 79 (76.7\%) had no residual cancer. Of the 118 managed endoscopically, none had residual cancer on follow-up endoscopy. The 21 (5.4\%) Dukes' C cancers were significantly associated with Kikuchi SM3/Haggitt 4 lesions $\left(\chi^{2}=10.85, p=0.005\right)$ and lesions with an involved/unsure excision margin $\left(\chi^{2}=7.44, p=0.017\right)$. Positive predictors of finding residual tumour at surgery were Kikuchi SM3/Haggitt Level 4 $\left(\chi^{2}=17.07, p<0.001\right)$, and any involved/unsure excision margin $\left(\chi^{2}=20.45, p<0.001\right)$. An excision margin $>0 \mathrm{~mm}$ was significantly associated with finding no residual tumour $\left(\chi^{2}=25.21, p<0.001\right)$. There was no difference in survival between surgical and endoscopic management $\left(\chi^{2}=0.634, p=0.426\right)$.

Conclusion Endoscopic management of a subgroup of MCPs appears safe and effective. A clear resection margin $(>0 \mathrm{~mm})$ appears sufficient to avoid surgery. Advanced lesions (Kikuchi 3/Haggitt 4) have a greater risk of residual cancer at surgery, and of lymph node metastases

Competing interests None declared

\section{PWE-091 MONITORING FAECAL OCCULT BLOOD TEST POSITIVITY IN THE NHS BOWEL CANCER SCREENING PROGRAMME}

doi:10.1136/gutjnl-2012-302514d.91

C Burtonwood, P Butler, M Young,* S Halloran. NHS Bowel Cancer Screening Programme, Southern Hub, Guildford, UK

Introduction The guaiac-based faecal occult blood test (gFOBt) used by the NHS Bowel Cancer Screening Programme relies on subjective visual assessment of colour change to determine positivity. The Southern Hub, one of five Programme Hubs in England, serves a total population of about 14.4 million people and handles about one million gFOBt kits every year.

Methods Test kit readers are tested for colour blindness and visual acuity before structured training and a period of supervision. Consistent test positivity within predefined limits is a quality measure of test kit readers and their performance is subject to weekly scrutiny. The percentage of positive test spots (six per test kit) is recorded weekly for every reader who completes $>100$ kits. The acceptable spot positivity range has been fixed at between $1.0 \%$ and $4.0 \%$, based on an approximation of \pm 2 SDs from the mean percentage positivity for all Hub staff over a rolling 6-month time period.

Results Screening Hubs have noticed characteristic positivity patterns among readers and this reflects the subjective nature of the measurement system. The data challenge Hubs to investigate idiosyncratic test kit reading habits of staff, to modify behaviour as indicated and monitor anticipated improvements in reading performance. The screening algorithm adopted in England results in approximately $85 \%$ of screen-positive participants completing two or three test kits. While this minimises the impact of individual readers, it adds complexity to the analysis of reader positivity. Repeat kits have a higher positivity and can bias reader weekly mean positivity rates. For all readers with high weekly positivity the proportion of second and third kits read forms part of the weekly assessment

Conclusion The Southern Hub has monitored reader positivity since August 2010 and the results are encouraging, with a reduction in reader imprecision and outliers. Initially, there was some resistance to monitoring positivity rates and the fear of being an outlier has the potential to lead staff to inappropriately modify their kit reading behaviour. New staff attend training sessions to learn about the concept of reader positivity and the interventions that may be put in place if their positivity falls outside the acceptable range. The process has now been widely accepted and the number of occasions that positivity falls outside this range per month is used as a key performance indicator.

Competing interests None declared.

\section{PWE-092 WILL THE NATIONAL AWARENESS AND EARLY DIAGNOSIS INITIATIVE (NAEDI) HAVE AN IMPACT ON BOWEL CANCER SCREENING ACTIVITY?}

doi:10.1136/gutjnl-2012-302514d.92

J Snowball, M Young,* S Halloran. NHS Bowel Cancer Screening Programme, Southern Hub, Guildford, UK

Introduction 1- and 5-year survival from all cancers in England is poorer than for other comparable countries, largely because of delayed diagnosis. The UK's Department of Health has estimated that if cancer survival in England could be improved to match the best in Europe, then 10000 lives would be saved every year, about 1700 of which would be from bowel cancer. The National Awareness and Early Diagnosis Initiative (NAEDI) is a Government plan to raise public awareness of the early signs and symptoms of cancer and, as one of the leading causes of cancer death, bowel cancer has been identified as a particular target. During 7 weeks in early 2011, a pilot bowel cancer NAEDI campaign was run in two Strategic Health Authorities (SHAs), including the South West SHA, with widespread coverage on local television, radio and newspapers and distribution of educational literature to general practitioners (GPs). The public was urged to consult their GP if they had any symptoms of bowel cancer and, consequently, attendance with relevant symptoms at GP practices increased by $48 \%$ with a $32 \%$ increase in 2 -week wait referrals. Little direct reference was made to the Bowel Cancer Screening Programme (BCSP).

Methods The BCSP Southern Hub has analysed screening data for the South West SHA to explore the indirect effects of the pilot campaign on screening activity. Screening data for the general practices covered by the media campaign were compared with data for the same practices a year earlier and with data for practices served by the Southern Hub not targeted during the campaign period at that time and a year earlier.

Results There was a small increase in overall uptake among individuals who were participating for the first time, but no evidence of a change in uptake by individuals who had previously participated in the Screening Programme. The Hub saw no increase in the number of calls received by the Helpline, test kits were not returned any more quickly and there was no change in the proportion of positive test kits. Comparisons drawn between the practices described are limited, however, by the likelihood that publicity 
reached areas not targeted for media coverage, the pilot campaign ran after Christmas when fluctuations in screening activity are considerable and age-extension was underway in some areas.

Conclusion At the end of January 2012, the Government launched a 9 -week national bowel cancer awareness campaign. Providers have been urged to plan for a $50 \%$ increase in GP referrals during the campaign and for a sustained increase in colonoscopy demand over the next 5 years. This analysis of local screening activity during the pilot campaign, however, suggests that the direct effect of the national NAEDI campaign on bowel cancer screening hub activity is likely to be modest.

Competing interests None declared.

\section{PWE-093 THE NHS BOWEl CANCER SCREENING PROGRAMME, SOUTHERN HUB-SCREENING ACTIVITY AND OUTCOMES}

doi:10.1136/gutjnl-2012-302514d.93

H Seaman, M Young, ${ }^{*}$ S Halloran. NHS Bowel Cancer Screening Programme, Southern Hub, Guildford, UK

Introduction As part of the NHS Bowel Cancer Screening Programme (BCSP) in England, every man and woman registered with a GP, living in England and aged 60-74 years, is invited to take part in screening every 2 years. The BCSP Southern Hub, hosted by the Royal Surrey County Hospital and one of five Hubs in England, serves a total population of about 14.4 million people and manages the screening activity in the south of England (excluding London). The Southern Hub handles nearly one million gFOB test kits every year. Here we provide a high-level overview of screening activity and outcomes for the Southern Hub since the Programme's launch in 2006.

Methods Screening invitees are sent a guaiac-based faecal occult blood (gFOB) test kit and asked to provide a faecal sample. Test kits are returned to the Hub for analysis. Participants with a positive ("abnormal") test are referred to a Specialist Screening Practitioner (SSP) for further assessment and investigation (usually colonoscopy) at one of 17 Screening Centres. All screening activity, including invitation uptake, gFOB test results, SSP referrals and colonoscopy outcomes are stored on a dedicated database-the Bowel Cancer Screening System (BCSS). The BCSS provides a rich source of data for observational analysis.

Results The uptake of screening invitations (the proportion of invitees that was adequately screened) is approximately $56 \%$ overall. Uptake is generally higher for women ( $61 \%$ vs $55 \%$ ), although improves with age in men. The proportion of positive test kits ("positivity") is higher for men (2.6\%) than for women (1.6\%) at all ages. The number of colonoscopies performed at the Screening Centres has increased over time. About $40 \%$ of the screened population that tests positive and undergoes colonoscopy has significant neoplasia (cancer, high- or intermediate-risk adenomas). The prevalence of significant neoplasia is greater in men and increases with age. The proportion of significant neoplasia detected in screening episode 2 is lower than in episode 1, reflecting successful detection of lesions in the first episode.

Conclusion The BCSS data are encouraging and indicate that the BCSP in England is likely to achieve its goal of reducing mortality from bowel cancer.

Competing interests None declared.

\section{PWE-094 UNDERSTANDING NON-PARTICIPATION IN BOWEL CANCER SCREENING: A QUALITATIVE STUDY}

doi:10.1136/gutjnl-2012-302514d.94

${ }^{1} \mathrm{~N}$ J Hall, ${ }^{*} \mathrm{G}$ P Rubin, ${ }^{2} \mathrm{D}$ Weller, ${ }^{3} \mathrm{~J}$ Wardle, ${ }^{1} \mathrm{C}$ Dobson, ${ }^{4} \mathrm{M}$ Ritchie, ${ }^{1,5,6} \mathrm{C}$ Rees.

${ }^{1}$ School of Medicine and Health, Durham University, Stockton, UK; ${ }^{2}$ Community Health
Sciences, University of Edinburgh, Edinburgh, UK; ${ }^{3}$ Health Behaviour Unit, University College London, London, UK; ${ }^{4}$ South of Tyne Bowel Cancer Screening Centre, Queen Elizabeth General Hospital, Gateshead, UK; ${ }^{5}$ Gastroenterology, South Tyneside NHS Foundation Trust, South Shields, UK; ${ }^{6}$ Northern Region Endoscopy Group, UK

Introduction Uptake of the national bowel cancer screening programme (BCSP), at $52 \%$, needs to be improved or at least maintained if the screening programme is to achieve projected reductions in mortality and morbidity. Understanding the origins of non-participation is therefore important. This study used qualitative methods to explore the beliefs and experiences of individuals who had not responded either to their screening invitation or reminder.

Methods In-depth qualitative interviews with volunteers were used to enable maximum opportunity for exploration and inductive hypothesis generation. Non-participation was defined as having refused all of the invitations and reminders for FOB test screening received from the North East Hub of the BCSP at the time of contact. Interviewees were purposefully sampled to allow for diversity in terms of gender, geographical location and socioeconomic status. Data collection and analysis were carried out using strategies consistent with the principles of grounded theory with an emphasis on the constant comparison method. Data collection and analysis took place concurrently and continued until saturation (27 interviews)

Results The interviews provided an in-depth understanding of a range of reasons and circumstances surrounding non-participation, including contextual and environmental influences as well as factors specific to the screening test. The nature of the data also allowed an appreciation of the potential for changes in beliefs, awareness and intention over time. Most of the interviewees had positive attitudes towards the BCSP, even those who did not feel screening was appropriate for them or who did not wish to take part. Many had intended to take part or intended to take part in the future. The main emergent categories included: practicalities of screening, value of screening, knowledge and awareness, risk perceptions, intention, embarrassment, good "citizenship", guilt, control, and the influence of others.

Conclusion A range of different approaches may be required to improve uptake, depending on the experiences, circumstances, beliefs and existing levels of intention of non-participants. Many of the interviewees in this study reported an intention to take part in future screening rounds. This group might be responsive to repeat invitations, reminders, and aids to making the test practical. Individuals who are opposed to screening (or BCS in particular) may have been less willing to be interviewed. Research is needed to ascertain whether different groups of non-responders require different approaches to intervention.

Competing interests None declared.

\section{PWE-095 ROLE OF RESTRICTED FLUID THERAPY IN PATIENTS UNDERGOING LAPAROSCOPIC AND OPEN COLORECTAL SURGERY: A SYSTEMATIC REVIEW AND META-ANALYSIS OF PUBLISHED RANDOMISED CONTROLLED TRIALS}

doi:10.1136/gutjnl-2012-302514d.95

M Sajid, N Ladwa,* P Sains, M K Baig. Department of General and Laparoscopic Colorectal Surgery, Worthing Hospital, Worthing, UK

Introduction Sub-optimal fluid therapy during peri-operative time period may influence the postoperative mortality and morbidity. The aim of this article is to systematically review the randomised trials analysing the restricted fluid therapy (RFT) and non-restricted 